\title{
Insights into Mechanical and Thermal Properties of Additively Manufactured PLA Samples Triggered by Automotive Industry Demands
}

\author{
DANA LUCA MOTOC ${ }^{1 *}$, HORATIU TEODORESCU DRAGHICESCU ${ }^{2}$, DANIELA FLOREA ${ }^{1}$, \\ ION PREDA ${ }^{1}$, NICOLAE ISPAS ${ }^{1}$ \\ ${ }^{1}$ Transilvania University of Braşov, Faculty of Mechanical Engineering, Department of Automotive and Transport \\ Engineering, 1 Politehnicii Str., 500024, Brașov, Romania \\ ${ }^{2}$ Transilvania University of Brașov, Faculty of Mechanical Engineering, Department of Mechanical Engineering, 1 \\ Politehnicii Str., 500024, Brasov, Romania
}

Abstract: Paper explores the influence of the infill density (\%), as a process parameter in additive manufacturing (3D printing), upon the mechanical (tensile, impact) and thermo-physical properties of PLA samples. The results indicated degradation of both tensile strengths and moduli with decrease of samples' relative density from $100 \%$ to $25 \%$ with $49.9 \%$ and $42.0 \%$, respectively. Similar behaviour holds on impact strength values that degrades with $56.0 \%$ for the samples printed using a $25 \%$ infill density. The Young's modulus variation with relative density values was approximated using a $3^{\text {rd }}$ order polynomial function, in accordance with the expression for closed-cell thick edge rhombus cellular structures. All PLA samples revealed small difference on their coefficients of linear thermal expansion, irrespective of their infill densities, with a decrease of $6.34 \%$ in the lowest relative density value specimens, indicating enhanced stability within selected temperature range. Glass transition temperatures were approximately located at $65^{\circ} \mathrm{C}$ whereas cold-crystallization around $80^{\circ} \mathrm{C}$, thus unaffected by selected process parameter.

Keywords: polylactic acid (PLA), additive manufacturing, mechanical properties, thermal stability

\section{Introduction}

Nowadays' highly competitive automotive market demands solutions to address both fuel efficiency and emissions performance since OEMs (Original Equipment Manufacturers) are bounded by strictly environmental regulations, technological advances and customers' fads. Among different approaches, lightweight automotive design, especially for body-in-white (BIW) and powertrains, proved to be one of the most important solution adopted to address the previous issues. (Mallick, 2010; Pervaiz et al., 2016; Del Pero et al., 2017; Kumar Dama et al., 2018)[1-4]. Conversely, the electric vehicles do not need solutions for fuel efficiency and emission reduction. These are being constructed from a lightweighting perspective to compensate for their battery weights (Mayyas et al., 2017)[5].

Additive manufacturing technologies are increasingly spread across various industries, from medical to architecture and construction, prototyping and manufacturing to art and food (Ngo et al., 2018; Yan et al., 2018)[6, 7]. Education benefits most from advances in the area if accounting on the advantages, such as cost-effectiveness, flexibility in design, material savings and personal customization.

Since fused deposition modeling (FDM) was adopted as an additively technique for 3D printers, the correspondingly market offers various configurations, including fully and non-assembled open-sources as well as commercial systems. These 3D printers produce different geometries using fused-filament fabrication typically in polylactic acid (PLA) and acrylonitrile butadiene styrene (ABS) polymers and composites of thereof.

Numerous studies have been performed to investigate the modification of process parameters influence on mechanical properties of PLA based parts. In Lanzotti et al. (2015) study was reported the

\footnotetext{
*email: danaluca@unitbv.ro
} 
influence of layer thickness, infill orientation and number of shell perimeters on the mechanical properties [8]. An empirical model, which connects the process parameters and mechanical properties, was proposed and good degree of compliance with other data from literature was found. Contribution of Dobrescu et al. (2019) reported the tensile properties of PLA and PLA based composites (e.g. fillers copper, aluminium, graphene) accounting different infill densities (60\%, 80\% and 100\%, respectively) and raster angles $\left( \pm 45^{\circ}\right.$ and $\left.\pm 60^{\circ}\right)$ [9]. Research results pointed toward property's degradation for PLA based composites irrespective of filler type compared with the solid PLA specimens. Additionally, in Behzadnasab et al. (2019) study was examined the effects of printing temperature, pattern and annealing conditions on tensile strength and modulus of PLA samples [10]. Research results suggest the negative impact of the annealing process on the mechanical properties while the tensile strength increased with the printing temperature.

In relation with the herein subject, the study of Lubombo and Huneault (2018) further examined the effect of the infill patterns on the mechanical properties of lightweight 3D printed cellular PLA parts [11]. Their results revealed an increase in both stiffness and strength values by keeping the same infill density value but using different infill pattern (e.g. hexagonal, square, square-diagonal structures, etc.). Research group of Schmitt et al. focused on the lightweighting design for automotive industry focusing on the optimal infill configurations for both tensile and flexural configurations $(2019,2020)[12,13]$. A set of case studies, including racing wheel and control arm, accompanied their studies addressing the issue of reducing the component mass and printing time while deploying optimal infill configurations.

Recently, Aloyaydi et al. (2020) conducted low velocity impact and compression tests on PLA specimens' 3D printed at different infill patterns: triangle, grid, quarter-cubic and tri-hexagon [14]. Their findings have shown that the grid pattern produced the highest compressive strength while the triangular pattern exhibited the highest absorbed energy in impact tests.

Flexural and tensile behavior of PLA, ABS and PLA-ABS materials were reported by Dhinesh et al. (2020) [15]. They focus on the ultimate tensile and flexural strengths accounting various blend ratio of aforementioned at an infill density of 50\% for all specimens. Reported findings shown that the 50:50 mixture between PLA and ABS revealed the maximum flexural strength while the 80:20 the maximum tensile strength among all.

Literature survey reveals no focused interest on thermal properties of additively manufactured PLA samples, especially on their coefficients of thermal expansion. To the best of our knowledge, PLA specimens were selected as reference while comparing retrieved properties on PLA based composites. Thus, Pandis et al. (2019) conducted research on PLA specimens in contact with phase change materials (PCMs) to be used as encapsulation or tank material in thermal storage systems [16]. Deploying differential scanning calorimetry (DSC), they recorded the crystallization temperatures and enthalpies after immersing the $100 \%$ infilled PLA samples into selected PMCs in the liquid state. Their results revealed improvements on PLA's crystallinity over the selected time interval.

Sang et al. (2019) developed a PLA based composite reinforced with basalt short fibers as a potential 3D-printer feedstock [17]. They reported on thermal properties of developed additively printed specimens by DSC scans as well as on their thermal stability by thermal gravimetry measurements. PLA sample was selected as referential and no changes were recorded on glass transition temperature $\left(\mathrm{T}_{\mathrm{g}}\right)$ values.

A co-authored contribution of Ferri et al. explored the thermal expansivity and degradation properties of hydroxyapatite (HA) and $\beta$-tricalcium phosphate ( $\beta$ TCP) reinforced PLA composites (fillers wt.\% 10,20 and $30 \%$, respectively) for medical purposes [18]. Reported findings shown decrease in measured coefficients of linear thermal expansion with increase of the filler content, small discrepancies on $\mathrm{T}_{\mathrm{g}}$ values for $\beta$ TCP reinforced composites compared with the PLA reference but negative shifts in all coupons. The PLA specimens of this referred study were developed by injection moulding technique.

In line with previous, Yu et al. (2019) developed PLA/talc fused filaments and studied crystallinity degree of their combinations. The filler was deployed as a nucleating agent and its influence upon 
dynamic mechanical properties and melting behavior was studied and reported. Their findings underlined enhancements of dynamic mechanical properties of samples under study.

Since it goes beyond the purpose of this contribution to step insight into the mechanical and thermal properties of PLA materials from a review perspective, the previous is capturing the main idea of approaching a subject generously addressed. Thus, it can be noted that investigation on process parameters' influences upon these properties is not currently covering a broader range. Researchers focus mainly on infill density's values exceeding 50\% and samples' expansivity usually unaccounted.

The idea of conducting a study on mechanical and thermal properties of 3D printed PLA samples, as developed subsequently, serve twofold purposes. One regards with setting out an in-situ database for simulation and materials' development, while the other for further studies on injection-molded vs. additive printing comparison. The later is in relation with one of the aforementioned contributions [18].

The present study aims to provide the results of a systematically conducted research on FDM 3D printed PLA samples accounting different infill density values and asses the effects on the tensile, impact and thermal expansion properties. Novelty can be sought from research data consistency and their statistical processing that enables clear views on the material's properties trends while varying the selected process parameter. Further, expansivity of PLA samples over temperature range has been recorded to enable insights into the process parameters' influence upon glass temperature transitions and linear coefficient of thermal expansion values.

\section{Materials and methods}

\subsection{Material selection and sample preparation}

Polylactic acid (PLA) selected for this study was the $1.75 \mathrm{~mm}$ filaments $(750 \mathrm{~g})$ supplied by Ingeo $^{\mathrm{TM}}$ PLA 3D850 (NatureWorks LLC, USA). The PLA density is $1.24 \mathrm{~g} / \mathrm{cm}^{3}$ and the softening temperature about $85^{\circ} \mathrm{C}$ (ISO 75-04) in accordance with the technical data sheets from the supplier.

The Anycubic Chiron (Shenzhen, China) with $0.4 \mathrm{~mm}$ nozzle diameter was used to fabricate the PLA based samples for tensile, impact and thermal dilatometry tests. All samples were printed using the fused deposition modelling (FDM) technology under the following fixed process parameters: nozzle temperature $-210^{\circ} \mathrm{C}$, building platform temperature - current room environment, build orientation of front face - flat, printing velocity - $60 \mathrm{~mm} / \mathrm{s}$, layer height $-0.1 \mathrm{~mm}$, infill pattern deposition directions in alternate layers - raster angle $\pm 45^{\circ}$, infill pattern type - grid, percent infill density - 100, 75, 50 and $25 \%$, respectively. Samples were labelled function of their infill densities to easy addressing and data comparison.

Specimen geometry and dimensions were in accordance with the specifications of individual tests to be carried out. Sample type IV (115 mm x $19 \mathrm{~mm}$ x $3.2 \mathrm{~mm}$, dog shape type) was selected for tensile tests in compliance with ASTM D638-14, while for Izod impact tests the samples (76 mm x $19 \mathrm{~mm} \times 3.2 \mathrm{~mm}$ ) were printed in accordance to ASTM D256-18. Thermal dilatometry tests were requiring rectangular shaped samples with $25 \mathrm{~mm}$ x $5 \mathrm{~mm}$ x $5 \mathrm{~mm}$ dimensions, in accordance to ASTM D696-08.

Following, the parametric CAD files (*.stl) containing the samples' geometries were transferred to an open-source slicing software - CURA. The latter is to generate G-code files and to command and control the 3D printer for the fabrication of thereof.

Ten specimens, accounting different relative density values, were printed to be used in batches for the tensile tests. Alternately, the impact and thermal expansivity tests deployed five samples ensuring the properties' statistically significant differences.

\subsection{Material characterization - mechanical and thermo-physical properties}

Tensile strength and tensile modulus were retrieved using a LS100 universal machine from Lloyd Instruments (UK) with a load cell capacity of $100 \mathrm{kN}$. Additively manufactured samples were stretched to failure at a crosshead speed of $1 \mathrm{~mm} / \mathrm{min}$ in accordance to ASTM D638-14 standard and data recorded by Nexygen Plus ${ }^{\mathrm{TM}}$ acquisition software. Impact tests were performed on an Impact Galdabini (Italy) testing machine equipped with a pendulum powered at $150 \mathrm{~J}$, impact speed of $5.5 \mathrm{~m} / \mathrm{s}$ and Izod impact 
fins, respectively. Tests were carried out under normal indoor parameters - room temperature of $23 \pm 2{ }^{\circ} \mathrm{C}$ and relative humidity of $45 \pm 5 \%$.

Thermal expansion tests were performed on a differential dilatometer DIL 420 PC/1 (Netzsch GmbH, D), in accordance with ASTM E228-11 and DIN 53752-A standards: temperature mode - dynamic heating ramp, temperature range - from $25^{\circ} \mathrm{C}$ up to $100^{\circ} \mathrm{C}$, heating rate of $1 \mathrm{~K} / \mathrm{min}$, thermocouple - $\mathrm{S}$ type. Data acquisition was accomplished by means of the 64-bit Proteus ${ }^{\circledR}$ software package. The software includes semi-automatic routines for correction of the sample holder expansion, as well as, computation of the expansion coefficients, peak temperatures, inflection points, rate of expansion, etc.

\subsection{Statistical analysis}

Tensile, impact and thermal properties' mean values is being obtained using OriginPro ${ }^{\circledR} 2018$ built in descriptive statistics' functions. Additionally, one-way analysis of variance (ANOVA) followed by post hoc Tukey's comparisons between in pair's samples, is being performed at a significant level set to 0.05 .

\section{Results and discussions}

\subsection{Tensile and impact behaviour}

As mentioned previously, the specimens for tensile tests were stretched in batches of ten printed units for individual infill density selection, and the results averaged for the aimed property. Tensile properties, including Young's modulus, tensile strength and elongation at break were few of the main values retrieved from the stress-strain curves plotted in Figure 1 and Figure 2.

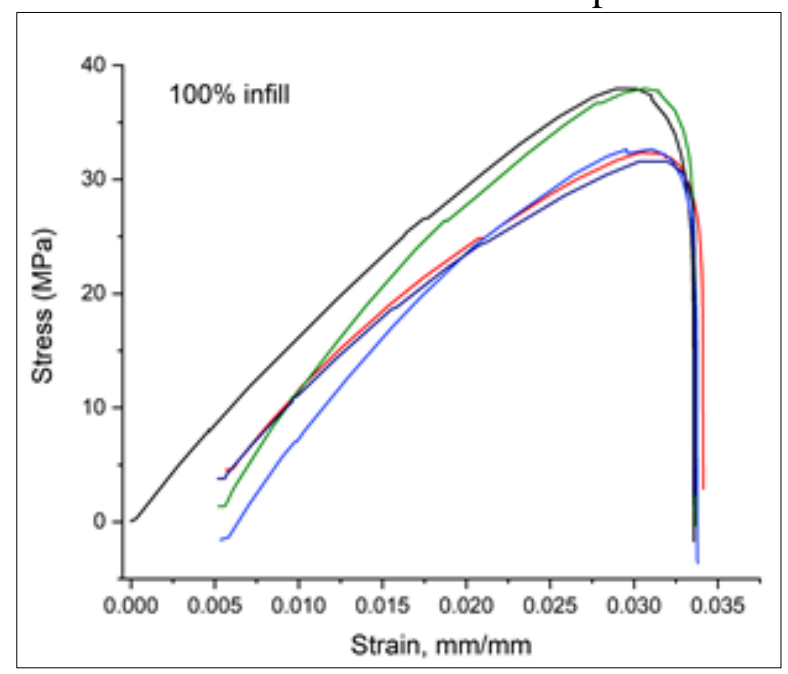

Figure 1. Excerpts of stress-strain curves of solid PLA (100\% infill density) samples

Stress-strain curve shapes are similar to those reported in literature for 3D printed PLA specimens subjected to tensile loading [19-21]. Consequently, structures additively manufactured at small infill densities are prone to degradation under mechanical loadings and thus unsuitable for automotive components. Exception may be sought for decorative elements and/or small auxiliary components that are not being subjected to loading conditions. Furthermore, all specimens experience a sudden brittle fracture of their inner cellular structure, with prolonged evolution until the fracture of their external shell, as plotted in Figure 2.

As it can be seen from above figures, the linear evolution of the tensile stress-strain results in various slopes and thus different effective Young's moduli, as plotted in Figure 3. The latter reduces with 22\%, 40 and $42 \%$, respectively compared with the solid parts as the infill densities diminished toward low values (i.e. from 100 to $75 \%, 50$ and $25 \%$, respectively).

The evolution of 3D printed samples' Young's modulus in tensile with the imposed infill density can be fitted at an R-squared (i.e. coefficient of determination) value of 1 with a $3^{\text {rd }}$ order polynomial function as provided in the expression below Table 1. This approximation function was proven to hold for closedcell thick edge rhombus cellular structures as herein, by Zhang et al., being more appropriate than the currently deployed expressions of Gibson and Ashby [22, 23]. 


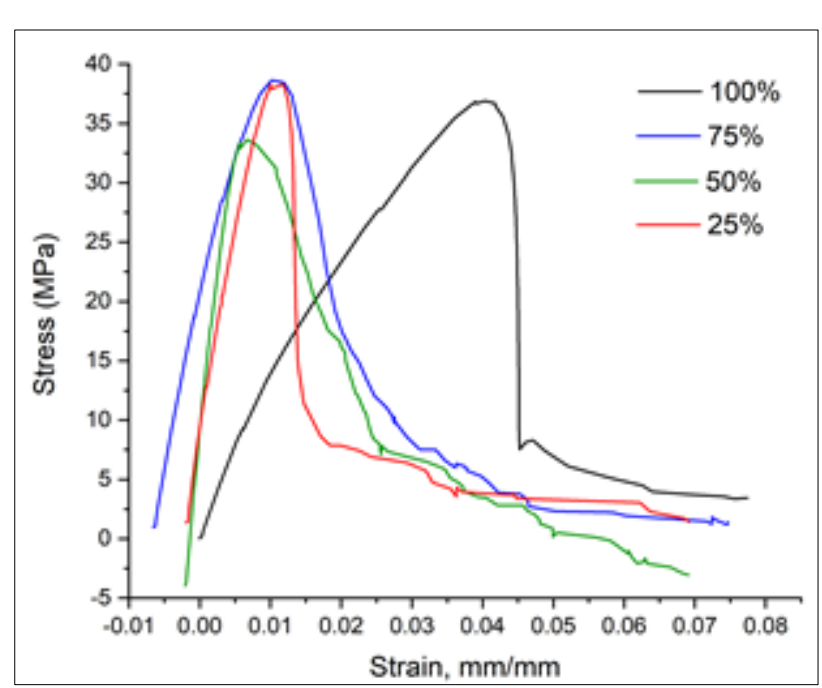

Figure 2. Excerpts of stress-strain curves accounting different relative density values

Thus, for the PLA in this study, the relative density variation function of relative density (i.e. percent infill density) has the following form:

$$
\frac{E}{E_{100 \%}}=-0.0028\left(\frac{\rho}{\rho_{100 \%}}\right)^{3}+0.68\left(\frac{\rho}{\rho_{100 \%}}\right)^{2}-37.42 \frac{\rho}{\rho_{100 \%}}+1753.5
$$

In the above relationship between relative density and relative elastic modulus $E$ is the Young's modulus of current sample, $\mathrm{E}_{100 \%}$ the Young's modulus of the solid sample (labelled herein as 100\%, selected as a reference sample), $\rho$ is the current sample's density and $\rho_{100 \%}$ are the solid PLA sample's density, respectively. Further insights recall the significance of the infill density as relative density ratio values expressed as percentage (\%).

One-way ANOVA statistics followed by a post-hoc Tukey mean comparison (at a 0.05 significance level) lead to cancellation of the null hypothesis $(\mathrm{F}=97.92)$. Thus, there are significant differences between the Young's modulus mean values while comparing in-pair samples. An exception holds in case of $50-25 \%$ pair. In-pair comparison on tensile strength values accounting on the percent infill density values show significant differences between population means at the 0.05 level $(\mathrm{F}=238.20)$. Alternative hypothesis is valid for impact strength mean comparison as well, at the 0.05 level revealing significant differences while running the post-hoc Tukey test $(\mathrm{F}=9.54)$.

Table 1 lists the percent strain at break and stress at break mean values. A decreasing trend with infill density compared to the solid sample reference hold for these listed tensile properties. As for the tensile and impact strengths' mean values, as plotted in Figure 4, degrades as the samples become less stiff, i.e. relative density diminishes.

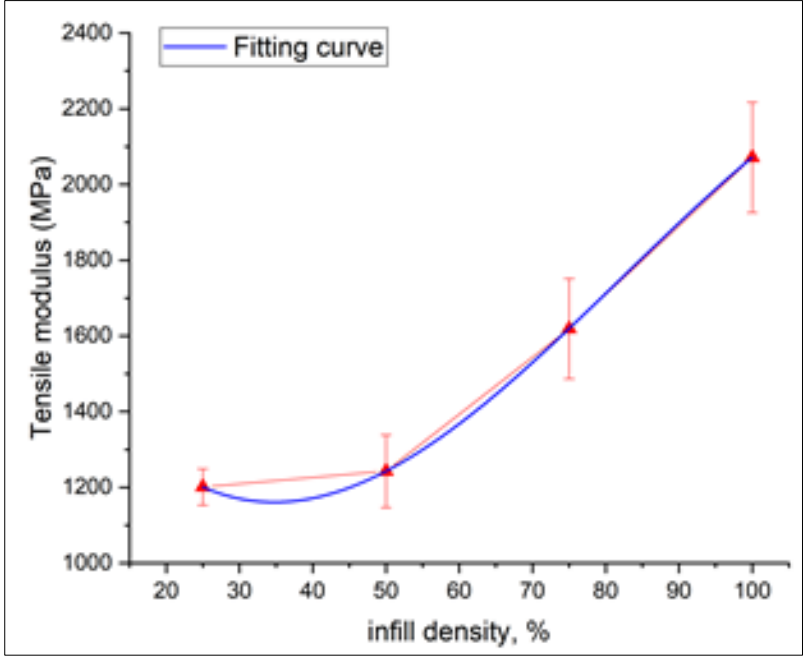

Mater. Plast., 57 (4), 2020, 193-201
Figure 3. Tensile Young's modulus evolution with infill density and its fitting curve 
Table 1. Supplementary tensile properties for PLA samples

\begin{tabular}{|c|c|c|c|}
\hline Sample ID & $\begin{array}{c}\text { Strain at break } \\
(\boldsymbol{\%})\end{array}$ & $\begin{array}{c}\text { Stress at break } \\
(\mathbf{M P a})\end{array}$ & Load at break (kN) \\
\hline $25 \%$ & $3.32 \pm 0.21$ & $12.503 \pm 0.98$ & $0.2914 \pm 0.02$ \\
\hline $50 \%$ & $3.63 \pm 0.19$ & $18.678 \pm 1.02$ & $0.3557 \pm 0.05$ \\
\hline $75 \%$ & $3.92 \pm 0.43$ & $24.749 \pm 1.34$ & $0.4797 \pm 0.04$ \\
\hline $100 \%$ & $3.99 \pm 0.56$ & $26.293 \pm 1.74$ & $0.5963 \pm 0.08$ \\
\hline
\end{tabular}

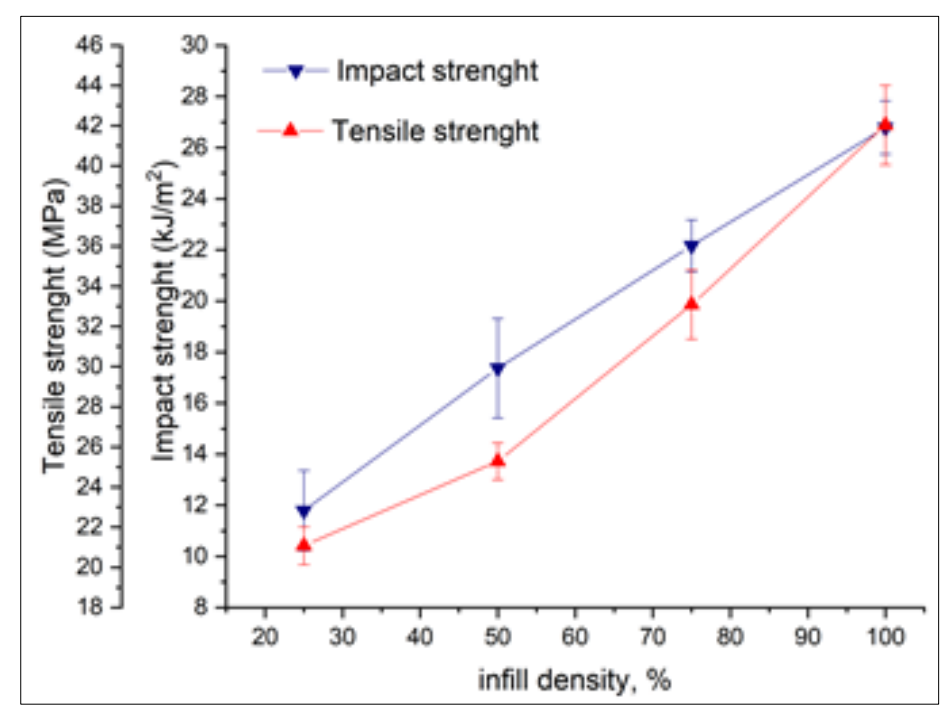

Figure 4. Tensile and impact strengths response to various infill density

The relative error values for tensile strengths are as follows - 21.3, 39.9 and $49.9 \%$ whereas for impact strengths - 17.3,35.2 and 56.0\%, respectively as the infill density values are decreasing compared to the solid sample. Roughly, at a $25 \%$ infill density (i.e. relative density ratio of 0.25 ) the mechanical property under debate degrades at half. This finding is important during prototyping processes of automotive parts, but not restricted to these, while focusing on financial issues rather than effective material properties.

\subsection{Thermo-physical behaviour}

Expansivity behaviour of materials deployed in FDM 3D printing processes is a critical factor since it greatly influences their dimensional stability. Accounting these circumstances, in herein study the linear coefficients of thermal expansion of PLA printed specimens with different infill densities were examined from room temperature up to $100^{\circ} \mathrm{C}$. In addition, the lowest value of $0.5 \mathrm{~N}$ was selected for the pushing-rods force against the samples to avoid their deformation with temperature increases.

As it can be seen in Figure 5(a) and (c), and other thermal parameters listed in Table 2 the expansion curves shapes are identical irrespective of samples infill density variation. Since a single material type was investigated, namely PLA, the results were as expected. Further insights reveal no shifts in the glass temperature values $\left(\mathrm{T}_{\mathrm{g}}\right)$ located at $65^{\circ} \mathrm{C}$ (with computed relative errors less than $0.75 \%$ ) for all samples irrespective of their relative densities. Since the outer shell was constant for all specimens irrespective of their infill densities, no further comments can be made with respect to this parameter. It can be considered for further developments along with other influencing factor's accounting samples' inner geometries. 


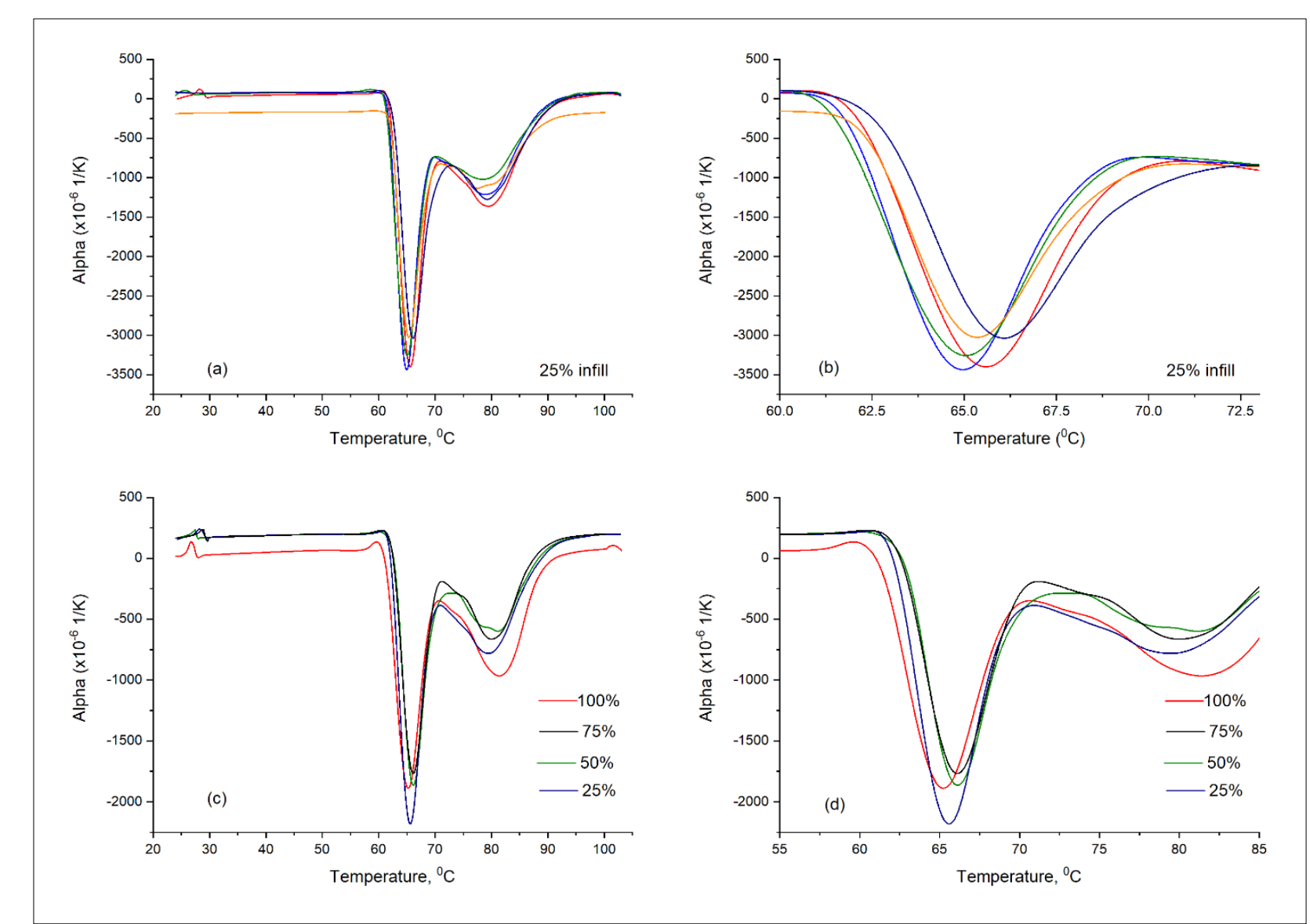

Figure 5. Linear coefficient of thermal expansion evolution with temperature: (a) 25\% infill PLA, (b) magnified view $25 \%$ infill PLA, (c) excerpts for $100 \%$ to $25 \%$ infill PLA samples and (d) magnified view for $100 \%$ to $25 \%$ infill PLA samples

Table 2. Thermal properties of PLA samples obtained from DIL curves

\begin{tabular}{|c|c|c|c|c|}
\hline Sample ID & $\mathbf{T}_{\mathrm{g}}\left({ }^{\circ} \mathbf{C}\right)$ & $\mathbf{T}_{\mathrm{cc}}\left({ }^{\circ} \mathbf{C}\right)$ & $\alpha_{\operatorname{Tg}} \times 10^{-3}(1 / K)$ & $\mathbf{T}_{\mathrm{g}}\left({ }^{\circ} \mathbf{C}\right)$ ref. \\
\hline $25 \%$ & $65.1 \pm 0.51$ & $79.58 \pm 0.43$ & $-4.01 \pm 3.27$ & \multirow{4}{*}{$\begin{array}{c}\text { PLA (solid): } \\
65 \text { [17]; } 62.3 \text { [18]; } 62.57 \text { [21]; } \\
64.8 \text { [24] }\end{array}$} \\
\hline $50 \%$ & $65.6 \pm 0.30$ & $80.10 \pm 0.25$ & $-3.45 \pm 2.86$ & \\
\hline $75 \%$ & $65.8 \pm 0.28$ & $80.94 \pm 0.13$ & $-3.01 \pm 2.65$ & \\
\hline $100 \%$ & $65.28 \pm 0.36$ & $81.12 \pm 0.20$ & $-4.01 \pm 3.27$ & \\
\hline
\end{tabular}

Compared with the reference data sheets of the PLA's supplier, there are small discrepancies between the $\mathrm{T}_{\mathrm{g}}$ values retrieved by DMA measurements occurred at $63^{\circ} \mathrm{C}$ but relatively large if retrieved by DSC measurements, occurred at $57^{\circ} \mathrm{C}$. Differences can be regarded mainly to the inherent properties of semicrystalline polymers generically known as PLA. Influences that come from the FDM 3D printing process parameters and sample infill patterns/dimensions as well as from measurements, devices deployed accounting differences on thermal loading conditions (e.g. thermal loading, temperature range, etc.) can be further accounted.

In the vicinity of $T_{g}$ points, PLA samples experiences abrupt chances in their thermal expansivities variation over temperature range caused by polymer chain mobility (see correspondingly values in Table 2). At these points, samples are softening and release high amounts of thermal stress inherited from the FDM 3D printing process.

Two peaks are encountered in the linear coefficient of thermal expansion $(\alpha)$ evolution with temperature curves. The first one corresponds to the glass transition temperature $\left(\mathrm{T}_{\mathrm{g}}\right)$ as mentioned above, while the second one can be regarded as cold-crystallization temperatures of PLA samples' $\left(\mathrm{T}_{\mathrm{cc}}\right)$. 
Table 2 lists the values of these temperatures. The small discrepancies between $\mathrm{T}_{\mathrm{cc}}$ values recorded at different infill density values, around $80^{\circ} \mathrm{C}$, should be regarded to the huge data amount to be processed proven the temperature step deployed (i.e. $1 \mathrm{~K} / \mathrm{min}$ ). Supplementary, Table 2 lists $\mathrm{T}_{\mathrm{g}}$ values from references to easy data comparison. PLA based structures should be used prior these $\mathrm{T}_{\mathrm{g}}$ values.

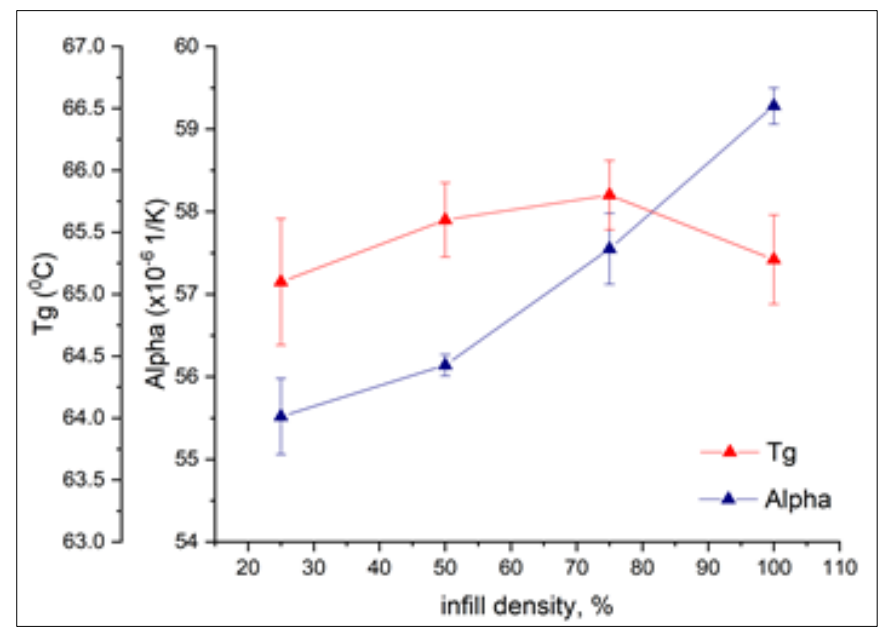

Figure 6. Mean linear coefficient of thermal expansion and glass temperature's variation with specimens infill densities

Additionally, coefficients of linear thermal expansion (CTE or $\alpha$ ) vary linearly within $25^{\circ} \mathrm{C}-50^{\circ} \mathrm{C}$ range (i.e. temperature use values) and their mean values at selected infill densities was plotted in Figure 6. As acknowledged, a decreasing tendency from the reference samples $\left(59.28 \cdot 10^{-6} \pm 0.221 / \mathrm{K}\right)$ to the samples with the lowest infill density $\left(55.52 \cdot 10^{-6} \pm 0.761 / \mathrm{K}\right)$ is being recorded, with the relative errors in the range: $2.92 \%$ (i.e. $75 \%$ infill), $5.30 \%$ (i.e. $50 \%$ infill) and $6.34 \%$ (i.e. $25 \%$ infill), respectively.

\section{Conclusions}

The current investigation examined the effects of infill density values upon the mechanical (tensile, impact) and thermo-physical properties of FDM 3D printed PLA samples for evaluating variation trends under statistically significant intervals. Properties' degradation with decrease on samples' relative densities was found on both tensile and impact strengths as well as on tensile moduli. These findings are important in weight and printing time reduction and thus related cost lowering at the pay-off of unloadable structures and decorative elements' automotive applications.

Rarely reported in literature stream for 3D printed structures, the coefficients of linear thermal expansion (CTE) were under scrutiny and relatively small variations from values recorded on the solid PLA specimens reported. Further, glass transition and cold-crystallization temperatures retrieved from CTE evolution were around 65 and $80^{\circ} \mathrm{C}$, respectively for all specimens irrespective of the percent infill densities selected. All 3D printed PLA samples exhibited stability under thermal loading within temperature use range. All results are important to engineers and designers to use them with FEA simulations and/or to enable further scaling to production applications. Further investigations of 3D printed vs. injection moulded comparison represents an interesting and into near future approachable subject.

\section{References}

1.MALLICK, P.K., 5-Thermoplastics and thermoplastic-matrix composites for lightweight automotive structures, in Materials, Design and Manufacturing for Lightweight Vehicles, P.K. Mallick, Editor. 2010, Woodhead Publishing. p. 174-207.

2.PERVAIZ, M., et al., Emerging Trends in Automotive Lightweighting through Novel Composite Materials. Materials Sciences and Applications, 2016. 07: p. 26-38. 
3.DEL PERO, F., M. DELOGU, M. PIERINI, The effect of lightweighting in automotive LCA perspective: Estimation of mass-induced fuel consumption reduction for gasoline turbocharged vehicles. Journal of Cleaner Production, 2017. 154: p. 566-577.

4.KUMAR DAMA, K., V. SURESH BABU, R.N. RAO, State of the Art on Automotive Lightweight Body-in-White Design. Materials Today: Proceedings, 2018. 5(10, Part 1): p. 20966-20971.

5.MAYYAS, A., et al., Vehicle's lightweight design vs. electrification from life cycle assessment perspective. Journal of Cleaner Production, 2017. 167: p. 687-701.

6.NGO, T.D., et al., Additive manufacturing (3D printing): A review of materials, methods, applications and challenges. Composites Part B: Engineering, 2018. 143: p. 172-196.

7.YAN, Q., et al., A Review of 3D Printing Technology for Medical Applications. Engineering, 2018. 4(5): p. 729-742.

8.LANZOTTI, A., et al., The impact of process parameters on mechanical properties of parts fabricated in PLA with an open-source 3-D printer. Rapid Prototyping Journal, 2015. 21(5): p. 604-617.

9.DOBRESCU, T., et al., Tensile behaviour of PLA and PLA composite materials under different printing parameters. Mater. Plast., 56(4), 2019, 783-800.

10.BEHZADNASAB, M., et al., Effects of processing conditions on mechanical properties of PLA printed parts. Rapid Prototyping Journal, 2019. 26(2): p. 381-389.

11.LUBOMBO, C., M.A. HUNEAULT, Effect of infill patterns on the mechanical performance of lightweight 3D-printed cellular PLA parts. Materials Today Communications, 2018. 17: p. 214-228.

12.SCHMITT, M., R.M. MEHTA, I.Y. KIM, Additive Manufacturing Experimental Infill Testing and Optimization for Automotive Lightweighting. 2019, SAE International.

13.SCHMITT, M., M. MEHTA RAJ, Y. KIM Il, Additive manufacturing infill optimization for automotive 3D-printed ABS components. Rapid Prototyping Journal, 2020. 26(1): p. 89-99.

14.ALOYAYDI, B., S. SIVASANKARAN, A. MUSTAFA, Investigation of infill-patterns on mechanical response of 3D printed poly-lactic-acid. Polymer Testing, 2020. 87: p. 106557.

15.DHINESH, S.K., et al., Study on flexural and tensile behavior of PLA, ABS and PLA-ABS materials. Materials Today: Proceedings, 2020.

16.PANDIS, P.K., et al., Differential scanning calorimetry based evaluation of $3 D$ printed PLA for phase change materials encapsulation or as container material of heat storage tanks. Energy Procedia, 2019. 161: p. 429-437.

17.SANG, L., et al., Development of short basalt fiber reinforced polylactide composites and their feasible evaluation for $3 D$ printing applications. Composites Part B: Engineering, 2019. 164: p. 629639.

18.FERRI, J.M., et al., Thermal expansivity and degradation properties of PLA/HA and PLA/BTCP in vitro conditioned composites. Journal of Thermal Analysis and Calorimetry, 2019. 138(4): p. 2691-2702. 19.LETCHER, T., M. WAYTASHEK, Material Property Testing of 3D-Printed Specimen in PLA on an Entry-Level 3D Printer. Vol. 2. 2014.

20.DONG, Y., J. MILENTIS, A. PRAMANIK, Additive manufacturing of mechanical testing samples based on virgin poly (lactic acid) (PLA) and PLA/wood fibre composites. Advances in Manufacturing, 2018. 6(1): p. 71-82.

21.KUMAR, R., I. FARINA, On the 3D printing of recycled ABS, PLA and HIPS thermoplastics for structural applications. PSU Research Review, 2018. 2.

22.ZHANG, L.G., et al., Elastic Modulus of Rhombus Dodecahedron Closed-Cell Cellular Materials. Advanced Materials Research, 2012. 535-537: p. 1556-1559.

23.GIBSON, L.J., M.F. Ashby, Cellular Solids: Structure and Properties. 1999: Cambridge University Press.

24.YU, W., et al., Melt crystallization of PLA/Talc in fused filament fabrication. Materials \& Design, 2019. 182: p. 108013.

$\overline{\text { Manuscript received: } 19.08 .2020}$ 